\title{
Rod and Cone Contributions to Horizontal Cell Light Responses in the Mouse Retina
}

\author{
Jennifer Trümpler, ${ }^{1}$ Karin Dedek, ${ }^{1}$ Timm Schubert, ${ }^{1}$ Luis Pérez de Sevilla Müller, ${ }^{1}$ Mathias Seeliger, ${ }^{2}$ Peter Humphries, ${ }^{3}$ \\ Martin Biel, ${ }^{4}$ and Reto Weiler ${ }^{1}$ \\ ${ }^{1}$ Department of Neurobiology, Carl von Ossietzky University, D-26111 Oldenburg, Germany, ${ }^{2}$ Retinal Diagnostics Research Group, Department of \\ Ophthalmology II, Eberhard-Karls University, D-72076 Tübingen, Germany, ${ }^{3}$ Smurfit Institute of Genetics, Trinity College, Dublin 2, Ireland, and \\ ${ }^{4}$ Department Pharmazie, Ludwig Maximilians Universität, D-81377 Munich, Germany
}

\begin{abstract}
Mammalian B-type horizontal cells make contact with both photoreceptor types: the dendrites contact cone photoreceptors, whereas the axon terminal processes contact rods. Despite their distinct synaptic contacts, horizontal cell somata and axon terminals receive a mixture of rod and cone inputs. Interaction of the two photoreceptor systems is essential for adaptation of photoreceptor sensitivity to different levels of background illumination, and horizontal cells play a key role in this adaptation. In this study, we used transgenic mouse lines to examine the contributions of rod and cone photoreceptor inputs to horizontal cell light responses in the mouse retina: rod signals were isolated by recording intracellularly from horizontal cells in a mouse lacking the cone cyclic nucleotide-gated channel, which lacks cone function, and cone signals were assessed using the rhodopsin knock-out mouse, which is a model for pure cone function. We found that both horizontal cell compartments receive a mixture of inputs from both photoreceptor types. To determine whether these inputs arrive via the long axon connecting the compartments or by way of rod-cone gap junctional coupling, we assessed the rod and cone contributions to horizontal cell somatic and axon terminal light responses in the connexin36-deficient mouse retina, which lacks rodcone coupling. Our results confirm that rods and cones are coupled by connexin36, and suggest that signal transmission along the axon is unidirectional: signals are passed from horizontal cell soma to axon terminal but not from axon terminal to soma.
\end{abstract}

Key words: retina; horizontal cell; axon terminal; CNG; rhodopsin; connexin36

\section{Introduction}

Interaction between the rod and cone pathways is extensive: rods and cones are electrically coupled (Raviola and Gilula, 1973; Smith et al., 1986; Schneeweis and Schnapf, 1995; Krizaj et al., 1998; Tsukamoto et al., 2001), and rod signals are funneled into the cone pathways at several stages in visual processing (for review, see Völgyi et al., 2004). This interaction of the two photoreceptor systems is essential for adaptation of photoreceptor sensitivity to different levels of background illumination. Horizontal cells play a key role in this adaptation.

Mammalian B-type horizontal cells make synaptic contacts with both photoreceptor types: the dendrites contact cone photoreceptors, whereas the axon terminal processes contact rods (Kolb, 1970, 1974). The horizontal cell soma and axon terminal are connected by a long axon, which is thought to electrically isolate the two compartments. Nevertheless, rod inputs have been recorded in horizontal cell somata in the cat retina (Steinberg, 1969a,b,c; Nelson et al., 1975). These rod inputs differed in

Received Sept. 27, 2007; revised May 7, 2008; accepted May 11, 2008.

This work was supported by Deutsche Forschungsgemeinschaft WE 849 14-1 and the International Graduate School for Neurosensory Science and Systems. We thank Bettina Kewitz, Nicole Iben, and Dr. Edda Fahl for technical assistance with the transgenic mice.

Correspondence should be addressed to Dr. Reto Weiler, Department of Neurobiology, University of Oldenburg, P.0. Box 2503, D-26111 Oldenburg. Germany. E-mail: reto.weiler@uni-oldenburg.de.

DOI:10.1523/JNEUROSCI.1564-08.2008

Copyright $\odot 2008$ Society for Neuroscience $\quad$ 0270-6474/08/286818-08\$15.00/0 their sensitivity from rod inputs recorded from axon terminals; it was therefore argued that they could not be arriving at the soma via the horizontal cell axon and axon terminal (Nelson et al., 1975). In addition, Bloomfield and Miller (1982) maintained that if signals spread from axon terminal to soma, then a slit of light displaced across the retina should elicit two peaks of activity at the soma; this was not the case (Bloomfield and Miller, 1982). Thus, rod inputs are thought to reach the horizontal cell soma via rodcone gap junctions (Nelson et al., 1975). However, although both studies ruled out signal transmission from axon terminal to soma, the possibility of signal transmission from soma to axon terminal has never been examined.

This possibility has been difficult to test because of inadequate experimental procedures: previous studies distinguished rod from cone signals using stimuli of different wavelengths; however, this method provides only partial signal separation. In this study, we recorded pure rod and pure cone signals in horizontal cell somata and axon terminals using transgenic mice lacking either rod or cone light responses. Rod inputs were isolated by recording from horizontal cells in the CNGA3 knock-out mouse, which lacks cone function as a result of deletion of the conespecific $\alpha$ subunit of the cyclic nucleotide-gated channel (CNGA3) (Biel et al., 1999); and cone signals were assessed in the rhodopsin knock-out mouse, which is a model for pure cone function (Humphries et al., 1997; Jaissle et al., 2001). The contribution of rod-cone gap junctions to the transmission of rod and 

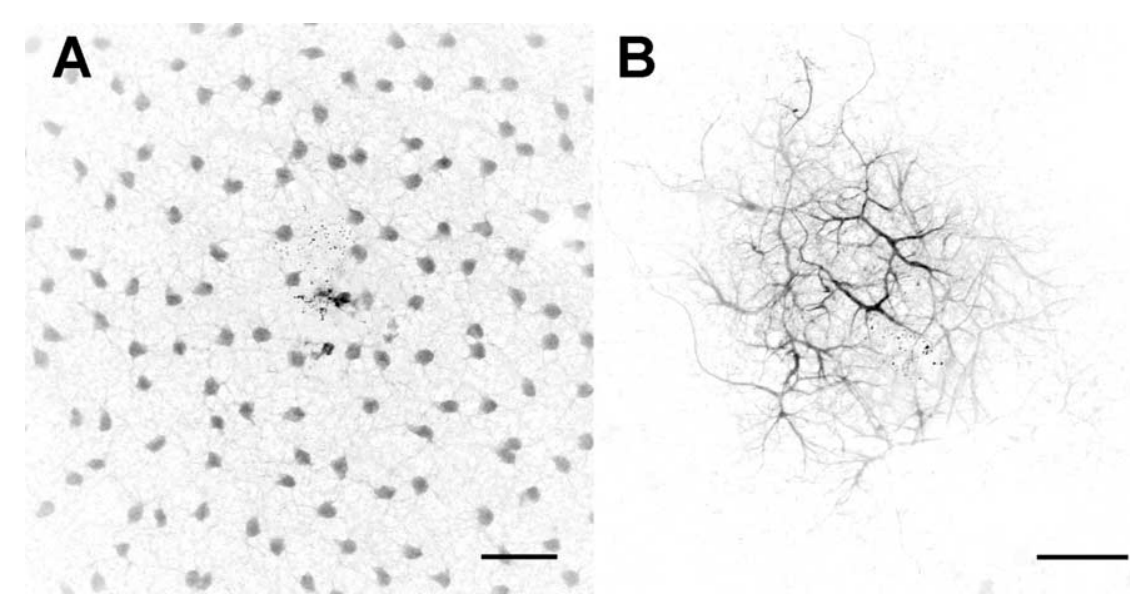

Figure 1. Morphological control. After intracellular recordings, horizontal cells were injected with Neurobiotin. $\boldsymbol{A}, \boldsymbol{B}$, Horizontal cell somata $(\boldsymbol{A})$ were easily distinguishable from axon terminals $(\boldsymbol{B})$. Scale bars, $50 \mu \mathrm{m}$.

cone signals to the horizontal cells was examined using connexin36 (Cx36)-deficient mice, in which rod-cone coupling is abolished (Deans et al., 2002).

We found that mouse horizontal cell somata and axon terminals respond to a mixture of rod and cone inputs. Recordings from connexin36-deficient mice indicated that signal transduction along the axon is unidirectional: cone signals are passed from horizontal cell soma to axon terminal.

\section{Materials and Methods}

Wild-type (C57BL/6 and 129/Sv), CNGA3 ${ }^{-1-}$ (129/Sv genetic background) (Biel et al., 1999), Cx36 ${ }^{-1-}$ (75\% C57BL/6 genetic background) (Güldenagel et al., 2001), and CNGA3 ${ }^{-1-} / \mathrm{Cx} 36^{-1-}$ mice $(129 / \mathrm{Sv}$ genetic background) aged 5 weeks to 3 months, and rhodopsin ${ }^{-1-}$ mice (C57BL/6 genetic background) (Humphries et al., 1997) aged 4-7 weeks, were used in these experiments. All experiments were performed in accordance with the institutional guidelines for animal welfare and the laws on animal experimentation issued by the German government. Careful attention was paid to the age of the mice, because photoreceptor deficiencies can cause degeneration of postsynaptic retinal neurons at later stages in development. For example, rhodopsin-deficient mice were used at 4-7 weeks of age because at this age, development is complete but cone degeneration has not begun, thus providing an all-cone retina (Jaissle et al., 2001).

Preparation for electrophysiology and intracellular recordings. Mice were housed under a $12 \mathrm{~h}$ light/dark cycle. Experiments were begun $6-7 \mathrm{~h}$ after the beginning of the light phase; mice were kept in a dark chamber for $18-19 \mathrm{~h}$ before being killed. The eye was enucleated in ice-cold Ringer's solution under dim red light. Three or four small incisions were made into the rim of the eyecup to facilitate inversion; the eyecup was then inverted onto a plasticine dome, stabilized with tissue paper, and transferred to the recording chamber. Ringer's solution, warmed to $28^{\circ} \mathrm{C}$ using a heated cannula (MultiChannel Systems), flowed continuously over the preparation from a small needle positioned at the apex of the inverted eyecup. The stabilizing tissue paper also served to drain the Ringer's solution and create a contact to the reference electrode. This configuration facilitated stable recordings lasting up to $1 \mathrm{~h}$. Ringer's solution contained (in mM) $117 \mathrm{NaCl}, 3 \mathrm{KCl}, 2 \mathrm{CaCl}_{2}, 1 \mathrm{MgSO}_{4}, 32$ $\mathrm{NaHCO}_{3}, 0.4 \mathrm{NaH}_{2} \mathrm{PO}_{4}, 15$ glucose, 0.1 glutamate, and 0.5 ascorbic acid, and was perfused with $95 \% \mathrm{O}_{2} / 5 \% \mathrm{CO}_{2}$ to maintain a $\mathrm{pH}$ of 7.4-7.5.

Light stimulation. After preparation, retinas were left to recover and dark adapt for $1 \mathrm{~h}$. Light stimuli were generated by a $100 \mathrm{~W}$ tungstenhalogen lamp and the intensity was controlled by a set of neutral density filters (Zeiss) to give a range of $7 \mathrm{log}$ units, with a maximum intensity of $0.32 \mathrm{~mW} / \mathrm{cm}^{2}$. For the experiments, full-field white light flashes of 250 $\mathrm{ms}$ duration were presented to the retina with interstimulus intervals of at least $10 \mathrm{~s}$.
Intracellular recordings. The procedure for intracellular recordings has been described (Shelley et al., 2006). Briefly, the membrane potential of the recorded cells was monitored intracellularly with glass microelectrodes (World Precision Instruments) pulled on a custommade horizontal puller and filled with 3\% Neurobiotin (Vector Laboratories) in $3 \mathrm{M} \mathrm{KCl}$. The electrodes had resistances of 80-120 M $\Omega$. The amplified signal was recorded with a PowerLab/ 4SP (ADInstruments) and stored on a computer for off-line analysis. Horizontal cells were identified by their large hyperpolarizing responses to light stimuli and by their depth in the retina. To prevent light adaptation, presentation of light flashes during the search for a cell was kept to a minimum; these test flashes had $\operatorname{dim}$ intensities $\left(<0.04 \mu \mathrm{W} / \mathrm{cm}^{2}\right)$, evoking responses smaller than $30 \%$ of the maximal response amplitude. Intensity-response profiles were derived from photoresponses elicited by full-field white light of increasing intensity; normalized response amplitudes were plotted against relative stimulus intensity. The profile of each cell was fitted with a sigmoidal function, from which the slope and the intensity eliciting half-maximal responses were derived.

After data collection, cells were injected with Neurobiotin by applying rectangular current pulses of $+1 \mathrm{nA}$ amplitude and $750 \mathrm{~ms}$ duration at $1 \mathrm{~s}$ intervals. Retinas were then removed from the pigment epithelium, fixed in $4 \%$ paraformaldehyde and incubated overnight in streptavidinindocarbocyanine (Cy3; Jackson ImmunoResearch) to label the tracer. Only cells successfully injected were accepted for evaluation. For labeling of cone pedicles, retinas containing injected cells were incubated with FITC-conjugated peanut agglutinin (diluted 1:16 in phosphate buffer containing $0.1 \%$ bovine serum; Vector Laboratories) overnight at $4^{\circ} \mathrm{C}$. Images were taken using a Leica TCS SL confocal microscope with a $40 \times$ or $63 \times$ oil-immersion objective, and adjusted using Adobe Photoshop 7.0 .

\section{Results}

We performed intracellular recordings from horizontal cells in the inverted eyecup preparation to examine the contributions of rod and cone inputs to horizontal cell somatic and axon terminal light responses in the mouse retina. Horizontal cells were identified by their large hyperpolarizing responses to light and their depth in the retina. Although horizontal cells could be reliably distinguished from other cell types by their light responses, somata and axon terminals could not be distinguished from one another in this way. Therefore, all recorded cells were filled with tracer for visual control (Fig. 1).

\section{Horizontal cell somata receive mixed rod-cone inputs}

Rod and cone contributions to horizontal cell light responses have been studied in detail in the cat retina (Steinberg 1969a,b,c). In general, the rod component of the horizontal cell response is slow, returning to baseline long after stimulus offset. Moderate intensities elicit a long-lasting decay after stimulus offset, referred to as the "rod after-effect" (Steinberg, 1969c; Niemeyer and Gouras, 1973). The cone component is faster and shows a square waveform matching stimulus duration (Steinberg, 1969b).

To provide appropriate controls for all of the transgenic mouse lines used in this study, we used two strains of wild-type mice: C57BL/6 and 129/Sv. All of the response characteristics described by Steinberg (1969a,b,c) and Niemeyer and Gouras (1973) could be identified in the light responses of C57BL/6 wildtype mouse horizontal cell somata (Fig. $2 \mathrm{~A}$ ), indicating that so- 

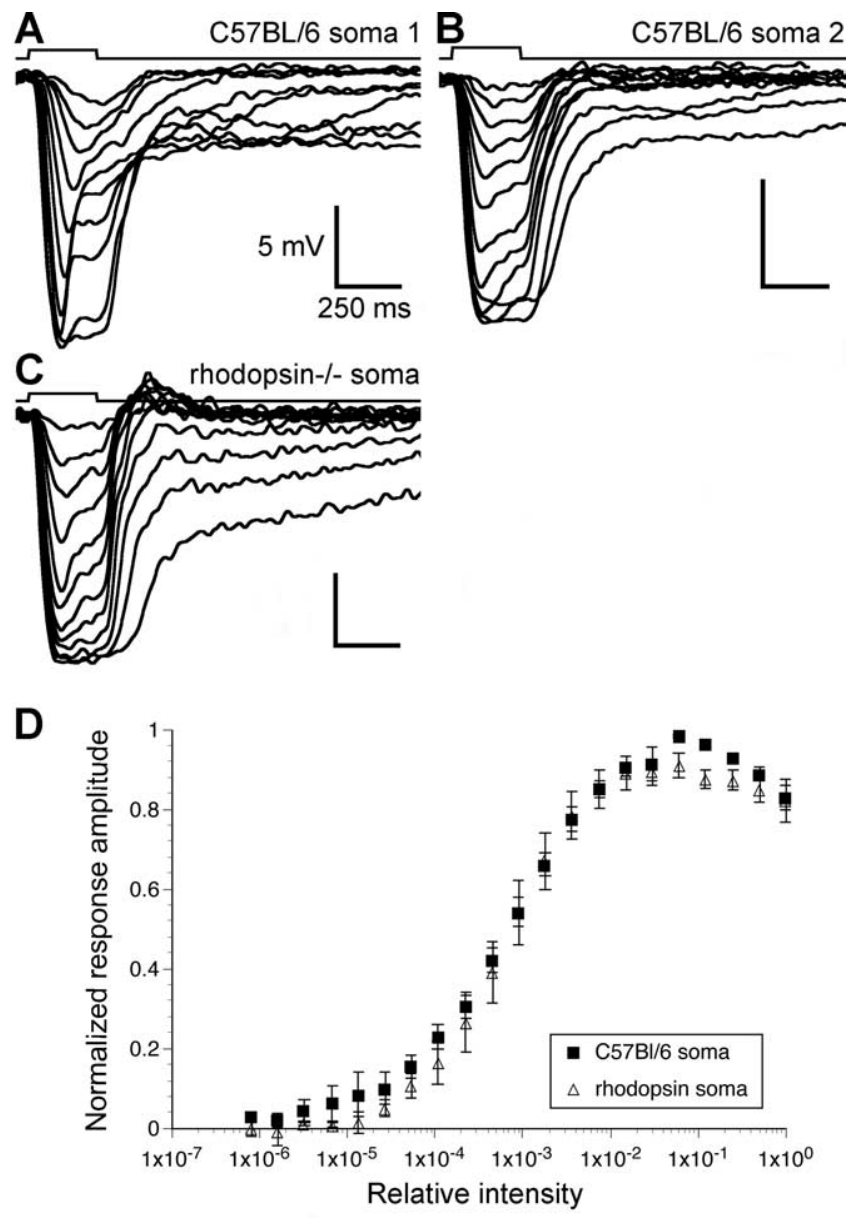

Figure 2. $\quad \boldsymbol{A}-\boldsymbol{C}$, Somatic light responses from $\mathrm{C57BL} / 6$ wild-type $(\boldsymbol{A}, \boldsymbol{B})$ and rhodopsindeficient retinas (C). Light stimuli consisted of full-field white light flashes of $250 \mathrm{~ms}$ duration. Increasing response amplitude reflects increasing stimulus intensity. $A, B$, C57BL/6 wild-type horizontal cell somata showed a range of response properties. The cell in $\boldsymbol{A}$ showed prominent rod components, including slow responses at low intensities and the rod after-effect at intermediate intensities. The cell in $\boldsymbol{B}$ showed primarily cone-dominated response properties. $\boldsymbol{C}$ Somata from rhodopsin-deficient mice showed exclusively cone-dominated response properties. A-C, Calibration: $5 \mathrm{mV}, 250 \mathrm{~ms}$. D, Normalized intensity-response profiles for C57BL/6 wild-type $(n=17)$ and rhodopsin-deficient horizontal cell somata $(n=12)$. Error bars indicate mean \pm SE. There was no significant difference in the intensity that elicited half-maximal responses in C57BL/6 wild-type and rhodopsin-deficient horizontal cell somata.

mata receive a mixture of rod and cone inputs. However, whereas some C57BL/6 horizontal cell somatic light responses had a prominent rod component, others had less pronounced rod contributions, or lacked visible rod signals entirely (Fig. 2 B). Thus, despite careful attention to the adaptational state of the retina (supplemental Fig. 1, available at www.jneurosci.org as supplemental material), C57BL/6 horizontal cell somata showed a wide range of response properties. Horizontal cell somata from the $129 /$ Sv strain showed much more consistent light responses; these cells always responded to light with a strong hyperpolarizing peak at light on, followed by a depolarizing sag and a slow return to baseline (Fig. 3A). The return to baseline was much slower in horizontal cells from $129 / \mathrm{Sv}$ mice than in cells from C57BL/6 mice.

Mouse rod and cone signals cannot be distinguished based on wavelength because the sensitivity range of the rods $\left(\lambda_{\max }=500\right.$ $\mathrm{nm})$ greatly overlaps with that of the middle-wavelengthsensitive cones $\left(\lambda_{\max }=510 \mathrm{~nm}\right)$ (Lyubarsky et al., 1999). Therefore, we used mouse models lacking either rod or cone function to elucidate the contributions of each photoreceptor type to wildtype horizontal cell light responses. In the rhodopsin-deficient retina, the rods cannot respond to light; thus, all light responses are purely cone generated. Conversely, in the CNGA3-deficient retina, the cones cannot respond to light; thus, all light responses are generated solely by the rods.

Figure $2 C$ shows traces recorded from a horizontal cell soma in a rhodopsin-deficient retina: as expected for a functionally rodless retina, the slow, rod-driven components were missing from the light responses. This was especially evident at low intensities, where the responses closely followed the stimulus time course. However, the high variability among C57BL/6 wild-type somata made distinction between somatic light responses of wild-type and rhodopsin-deficient retinas difficult. This variability was not seen among horizontal cell somata of rhodopsindeficient retinas: all light responses had the fast waveform typical of cone-dominated light responses $(n=12)$, and most (9 of 12 cells) showed the depolarizing rollback associated with feedback to the cones.

Conversely, in the CNGA3-deficient retina (Fig. 3B), the light responses were much slower, highlighting the slow characteristics of purely rod-driven responses. These response waveforms were rounded and lacked a depolarizing rollback. At moderate intensities, near the threshold for 129/Sv wild-type horizontal cell somata, somata from CNGA3-deficient retinas saturated, and their membrane potentials required several minutes to return to baseline. Higher intensities completely bleached the responses, and the membrane potential did not return to baseline for several tens of minutes (data not shown).

Comparing the intensity-response curves from these two transgenic mouse lines with those of the corresponding wild type gives an indication of the relative contributions of rods and cones to wild-type horizontal cell light responses (Figs. 2D, 3C). The intensities that elicit half-maximal responses in horizontal cell somata from rhodopsin-deficient retinas did not differ significantly from those from wild-type retinas (rhodopsin deficient, $I_{1 / 2}=2.3 \times 10^{-3} \pm 1.2 \times 10^{-3}$, mean $\pm \mathrm{SE}, n=12$; C57BL $/ 6$ wild type, $I_{1 / 2}=1.4 \times 10^{-3} \pm 0.5 \times 10^{-3}, n=17 ; p=0.14$, Student's $t$ test). However, horizontal cell somata from CNGA3deficient retinas showed a much higher sensitivity than 129/Sv wild-type horizontal cells, responding strongly to intensities that were at threshold level for wild-type horizontal cells. The intensities that elicit half-maximal responses in horizontal cell somata from CNGA-deficient retinas were significantly lower compared with the $129 / \mathrm{Sv}$ wild type (CNGA3-deficient, $I_{1 / 2}=3.5 \times 10^{-6} \pm$ $1.4 \times 10^{-6}, n=5 ; 129 / \mathrm{Sv}$ wild type, $I_{1 / 2}=0.5 \times 10^{-3} \pm 0.2 \times$ $\left.10^{-3}, n=8 ; p<0.05\right)$. This suggests that rod inputs to horizontal cell somata are strongly suppressed in the presence of cone inputs in wild-type retinas, as has been shown previously in amphibian horizontal cells in the mesopic state (Witkovsky et al., 1997).

Additional evidence supporting a mixture of rod and cone signals in wild-type horizontal cells can be derived from the slopes of the intensity-response curves. Where two photoreceptor types contribute to the horizontal cell responses, a shallow slope is expected because the combination of rods and cones leads to responses covering a wider range of intensities. Conversely, where only one photoreceptor type contributes, a steeper slope is expected. The mean intensity-response curve was significantly steeper for rhodopsin- $(1.08 \pm 0.10)$ and CNG-deficient horizontal cells $(1.01 \pm 0.06)$ than for the corresponding wild types $(\mathrm{C} 57 \mathrm{BL} / 6,0.84 \pm 0.04, p<0.01 ; 129 / \mathrm{Sv}, 0.68 \pm 0.08, p<0.01)$. Therefore, we can conclude that wild-type horizontal cell somata receive a mixture of rod and cone inputs. 

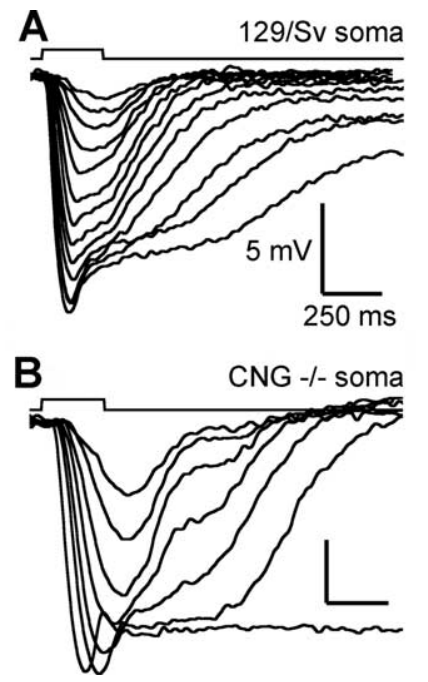

C

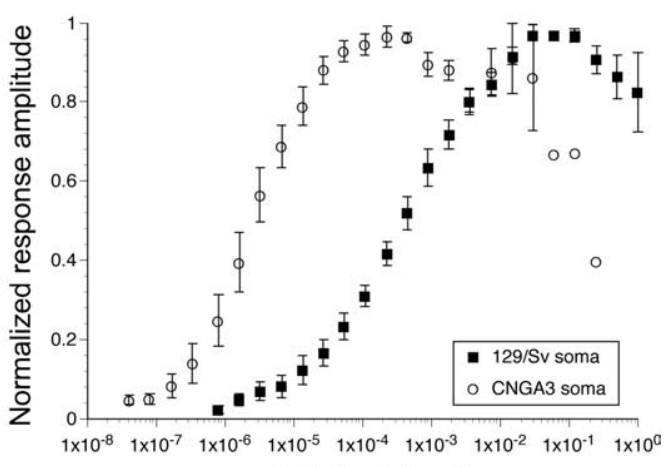

Relative intensity
Figure 3. Somatic light responses from 129/Sv and CNGA3-deficient retinas. Light stimuliare the same as in Figure 2.A, 129/Sv wild-type horizontal cell somata showed much more consistent light response properties than somata from C57BL/6 mice. $\boldsymbol{B}$, Somatic recordings from CNGA3-deficient retinas were easily distinguishable from the wild type. The square time course of the stimulus, visible in wild-type responses, is absent in responses from CNGA3-deficient retinas. $\boldsymbol{A}, \boldsymbol{B}$, Calibration: $5 \mathrm{mV}, 250 \mathrm{~ms}$. $\boldsymbol{C}$, Normalized intensity-response profiles for $129 / \mathrm{Sv}$ wild-type $(n=8)$ and CNGA3-deficient horizontal cell somata $(n=5)$. Error bars indicate mean \pm SE. Horizontal cell somata from CNGA3-deficient retinas responded to much lower light intensities than did $129 /$ Sv wild-type horizontal cells $(p<0.05)$. This huge shift in the intensity-response profile reflects the pure rod input to horizontal cells in the CNGA3-deficient retina. wild-type axon terminals. As was seen in the somatic recordings, horizontal cell axon terminals from CNGA3-deficient retinas responded to lower intensities than did wild-type axon terminals (Fig. 5C) (CNGA3 deficient, $I_{1 / 2}=1.9 \times 10^{-6} \pm$ $0.7 \times 10^{-6}, n=5 ; 129 / \mathrm{Sv}$ wild type, $I_{1 / 2}=$ $0.25 \times 10^{-6} \pm 0.18 \times 10^{-6}, n=5$ ); this difference was not significant.

As described above, additional evidence supporting a mixture of rod and cone signals in wild-type horizontal cells can be derived from the slopes of the intensity-response curves. There was no significant difference between the slopes of the intensity-response curves from rhodopsin-deficient $(0.92 \pm 0.03)$ and C57BL/6 wild-type horizontal cells $(0.92 \pm 0.14)$, but the intensity-response curves were significantly steeper for axon terminals in CNGA3-deficient retinas $(0.94 \pm 0.09)$ than for the $129 / \mathrm{Sv}$ wild type $(0.57 \pm 0.07 ; p<0.01)$. Therefore, we can conclude that wild-type horizontal cell axon terminals receive a mixture of rod and cone inputs, and the cone input strongly influences the sensitivity of the horizontal cell axon terminals.

\section{Horizontal cell axon terminals receive mixed rod-cone inputs}

Light responses of axon terminals were very similar to those of somata; axon terminal recordings were confirmed by visual control using tracer injection (Fig. 1). Like horizontal cell somata, C57BL/6 axon terminals also showed a mixture of rod and cone inputs and high variability between cells (Fig. 4A,B), whereas responses from 129/Sv retinas were more uniform (Fig. 5A). Although axon terminal recordings could not be distinguished from somatic recordings, axon terminals did show a higher sensitivity than somata (Fig. 6C), as has been reported previously (Suzuki and Pinto, 1986).

Rod and cone contributions to axon terminal light responses were also dissected by recording from axon terminals in CNGA3and rhodopsin-deficient retinas. The results were similar to those seen in somatic recordings. Figure $4 C$ shows traces recorded from a horizontal cell axon terminal in a rhodopsin-deficient retina: as was seen in the somatic recordings (Fig. 2C), the slow, rod-driven components were missing from the light responses. Conversely, in the CNGA3-deficient retina (Fig. 5B), the light responses did not reflect the square time course of the stimulus, as seen in the wild type, particularly at intermediate intensities. However, the strong similarity seen between axon terminals from 129/Sv and CNGA3-deficient retinas suggests a strong rod input to the axon terminal in the wild type.

Again, the intensity-response curves from these two transgenic mouse lines were compared with those of the appropriate wild type to examine the relative contributions of rods and cones to wild-type axon terminal light responses. The intensity-response curves of axon terminals from rhodopsin-deficient retinas were shifted to higher intensities compared with the wild type (Fig. 4D) (rhodopsin deficient, $I_{1 / 2}=1.3 \times 10^{-3} \pm 0.8 \times 10^{-3}$, $n=3$; C57BL/ 6 wild type, $I_{1 / 2}=0.3 \times 10^{-3} \pm 0.08 \times 10^{-3}, n=$ $7 ; p<0.05)$. Rod inputs therefore shape the response profile in

\section{How do rod inputs reach the soma?}

We reasoned that there are two possibilities: either rod signals spread to cones through rod-cone gap junctions, or they enter the horizontal cell axon terminal and spread along the axon to the soma. These pathways are not necessarily mutually exclusive. To address this issue, we used a mouse model in which rod-cone coupling is presumably abolished: the Cx36-deficient mouse (Güldenagel et al., 2001; Deans et al., 2002). We hypothesized that if rod signals reach the horizontal cell soma solely by way of rod-cone gap junctions, then somatic responses from Cx36deficient retinas should resemble the pure-cone responses from rhodopsin-deficient retinas.

Figure $6 \mathrm{~A}$ shows typical light responses of a horizontal cell soma from a Cx36-deficient mouse. These responses closely resemble the pure-cone responses obtained from rhodopsindeficient retinas. Their intensity-response profiles did not differ from those of the wild type (Fig. $6 C)\left(I_{1 / 2}=2.8 \times 10^{-3} \pm 1.2 \times\right.$ $\left.10^{-3} ; n=7\right)$. However, because the rod component of the somatic responses was so difficult to discern in the wild type, and therefore the somatic responses from rhodopsin-deficient (and Cx36-deficient) retinas closely resemble wild-type responses, a further step was needed to test our hypothesis.

We therefore attempted to record light responses from CNGA3/Cx36 double knock-out mouse retinas; these mice lack both cone responses and rod-cone gap junctions. We reasoned that if light responses could be obtained from horizontal cell somata in these mice, then these light responses must (1) originate in rods and (2) pass to the horizontal cell soma by way of the horizontal cell axon terminal and axon. We attempted these recordings on nine mice, and obtained a total of seven axon terminal recordings; no somatic recordings were obtained (Table 1). In our hands, wild-type retinas yield on average 17 somatic recordings for every seven axon terminals. Therefore, we conclude that the complete absence of somatic recordings in CNGA3/Cx36 


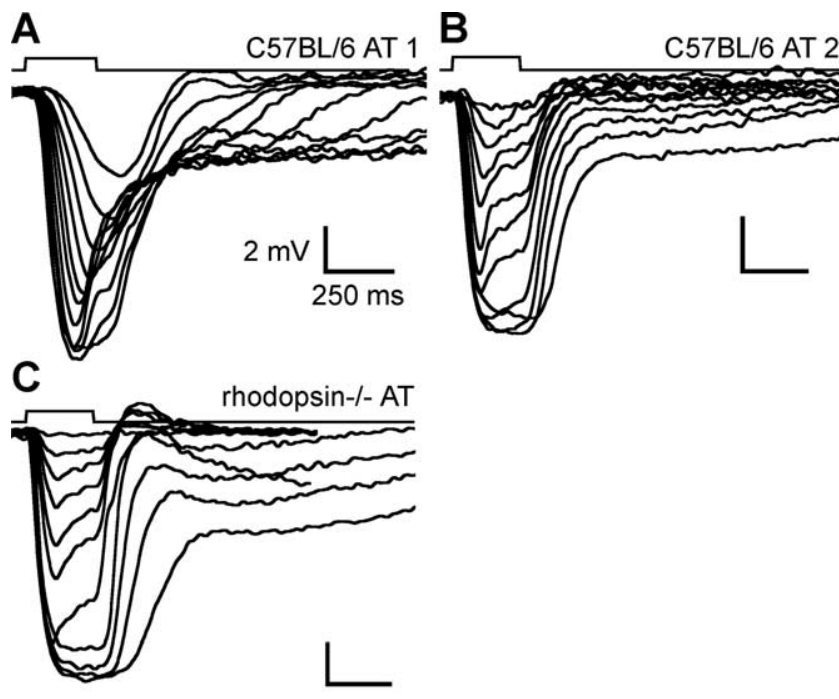

D

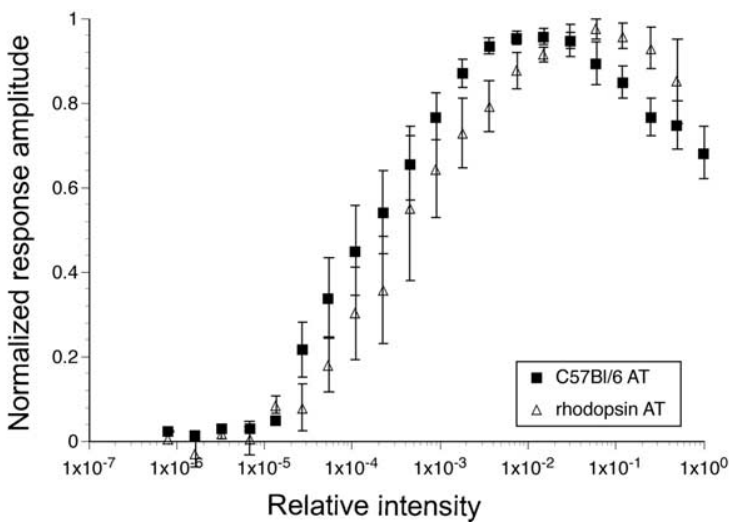

Figure 4. $A-C$, Axon terminal light responses from C57BL/6 wild-type $(A, B)$ and rhodopsindeficient retinas (C). Light stimuli are the same as in Figure 2.A, B, C57BL/6 wild-type horizontal cell axon terminals showed a range of response properties. The axon terminal in $\boldsymbol{A}$ showed prominent rod components, including slow responses at low intensities and the rod after-effect at intermediate intensities. The axon terminal in $\boldsymbol{B}$ showed primarily cone-dominated response properties. $C$, Axon terminals from rhodopsin-deficient mice showed exclusively conedominated response properties. $\boldsymbol{A}$-C, Calibration: $2 \mathrm{mV}, 250 \mathrm{~ms}$. D, Normalized intensityresponse profiles for $\mathrm{C} 57 \mathrm{BL} / 6$ wild-type $(n=7)$ and rhodopsin-deficient horizontal cell axon terminals $(n=3)$. Error bars indicate mean \pm SE. The intensity that elicited half-maximal responses in axon terminals was higher for rhodopsin-deficient retinas than for the wild type $(p<0.05)$.

double knock-out retinas is significant. It should be noted that the lack of somatic light responses was not a result of photoreceptor degeneration, because light responses were detected in axon terminals in CNGA3/Cx36 double knock-out retinas. These results support the theory of Nelson et al. (1975) that signals are not transmitted along the horizontal cell axon from axon terminal to soma. This also confirms the finding of Deans et al. (2002) that Cx36 forms gap junctions coupling the rods and cones in the mouse retina.

\section{How do cone inputs reach the axon terminal?}

The horizontal cell axon does not transmit signals from axon terminal to soma, but so far the possibility of signal transmission in the opposite direction has not been examined in the mammalian retina. To test whether cone signals reach the axon terminal via rod-cone coupling or by way of the horizontal cell soma and axon, we recorded light responses of horizontal cell axon terminals from Cx36-deficient retinas. We reasoned that if, in the wild type, cone signals reach the axon terminal by way of rod-cone gap junctions, then light responses of axon terminals from $\mathrm{Cx} 36$ deficient retinas should resemble those obtained from CNGA3deficient retinas, in which the cone component of the light responses was missing.

Light responses of an axon terminal from a Cx36-deficient mouse are shown in Figure $6 \mathrm{~B}$. These light responses closely resemble those obtained from somata from this mouse line, and are also very similar to axon terminal responses obtained from rhodopsin-deficient retinas (Fig. 4C), but quite distinctive from axon terminal responses obtained from CNGA3-deficient retinas (compare Fig. 5B). In particular, the responses in Cx36-deficient retinas show clearly the square time course of the stimulus at all intensities. This indicates a strong cone component in these axon terminal light responses, although the cones are no longer coupled to the rods. In addition, their intensity-response profiles did not differ from those of wild-type axon terminals (Fig. 6C) $\left(I_{1 / 2}=\right.$ $0.3 \times 10^{-3} \pm 0.2 \times 10^{-3}, \mathrm{Cx} 36$ deficient; $\left.n=3\right)$. It should be noted that a shift to lower intensities would be expected if the cone component were absent (compare Fig. $5 C$ ). Thus, rod-cone coupling is not necessary for spread of cone signals to horizontal cell axon terminals.

How, then, do cone signals reach the horizontal cell axon terminal? Axon terminals make synaptic contacts with rod photoreceptors in the wild-type retina. However, retinal neurons in transgenic mice with photoreceptor deficiencies have been shown to reorganize their synaptic contacts (Strettoi et al., 2002; Dick et al., 2003). To rule out the possibility that axon terminals in Cx36-deficient mice contact cone pedicles, we incubated retinas containing injected axon terminals with peanut agglutinin, which labels the base of the cone pedicles (Hack and Peichl, 1999; Haverkamp et al., 2001). Rotations of single scans revealed no overlap between axon terminal and cone pedicle labeling (Fig. 7) $(n=3)$. In addition, axon terminal morphology appeared normal; no evidence of sprouting or reorganization was seen. Thus, our data suggest that axon terminals receive cone inputs from the horizontal cell soma by way of the axon.

\section{Discussion}

Mouse horizontal cell somata receive synaptic inputs from cone photoreceptors, whereas their axon terminals receive synaptic inputs solely from rods. Despite this clear separation of synaptic inputs, both the somata and axon terminals of horizontal cells respond to a mixture of rod and cone inputs. Recordings from Cx36-deficient retinas indicated that rod signals reach horizontal cell somata via Cx36-containing gap junctions, thus confirming the role of Cx36 in rod-cone coupling. However, Cx36 was not required for conductance of cone signals to horizontal cell axon terminals. Our data suggest that transduction along the horizontal cell axon is unidirectional, from soma to axon terminal.

\section{Methodological considerations}

Rod signals in horizontal cells can be determined by the light response waveform of the horizontal cell, because the temporal properties of the rod response are not significantly filtered by signal transmission from photoreceptor to horizontal cell (Verweij et al., 1999). Because rod and cone signals cannot be easily dissected in the mouse retina using stimuli of different wavelengths, we took an alternative approach, using transgenic mouse lines: cone signals were assessed using the rhodopsin knock-out mouse, which is a model for pure cone function (Humphries et al., 1997; Jaissle et al., 2001); rod inputs were isolated in the CNGA3 knock-out mouse, which lacks cone function (Biel et al., 1999). This 

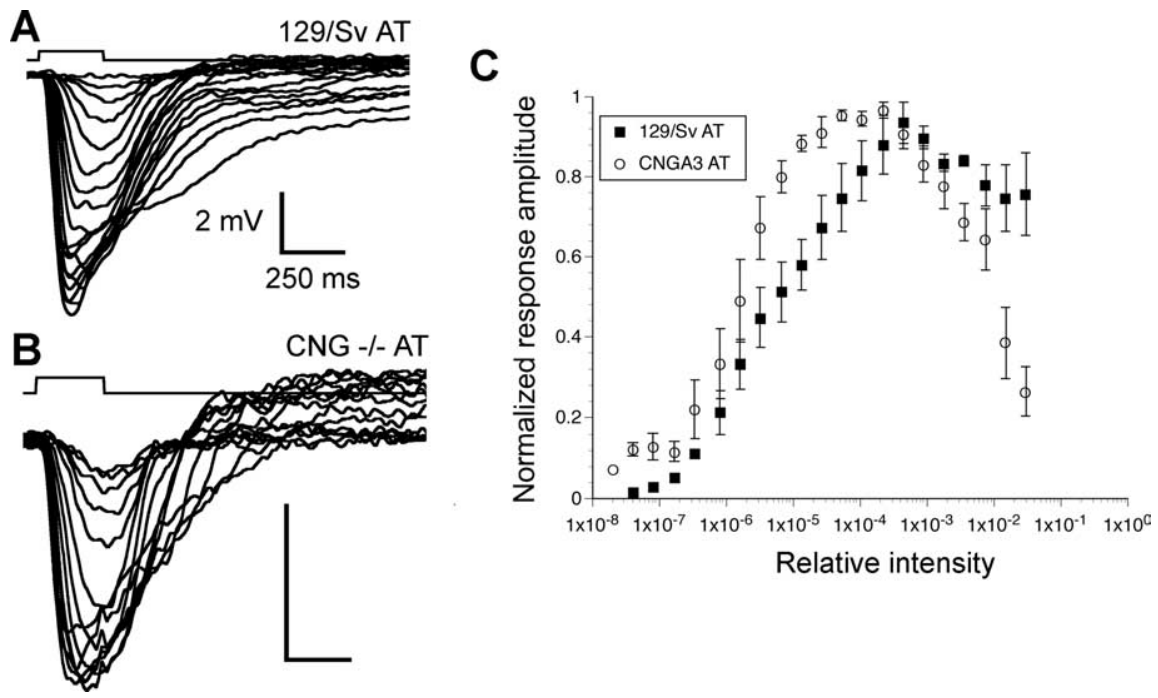

Figure 5. $\quad \boldsymbol{A}, \boldsymbol{B}$, Axon terminal light responses from $129 / \mathrm{Sv}$ wild-type $(\boldsymbol{A})$ and CNGA3-deficient retinas $(\boldsymbol{B})$. Light stimuli are the same as in Figure 2. Axon terminal recordings from CNGA3-deficient retinas were less distinguishable from the wild type, reflecting the strong rod input to these structures in the wild-type retina. $A, B$, Calibration: $2 \mathrm{mV}, 250 \mathrm{~ms}$. C, Normalized intensity-response profiles for 129/Sv wild-type $(n=5)$ and CNGA3-deficient horizontal cell axon terminals $(n=5)$. Error bars indicate mean \pm SE. The intensity that elicited half-maximal responses in axon terminals was lower for CNGA3-deficient retinas than for 129/Sv wild-type axon terminals; this difference was not significant. The response profiles of axon terminals from CNGA3-deficient retinas were significantly steeper than those from the wild type, confirming the mixture of inputs into wild-type axon terminals.
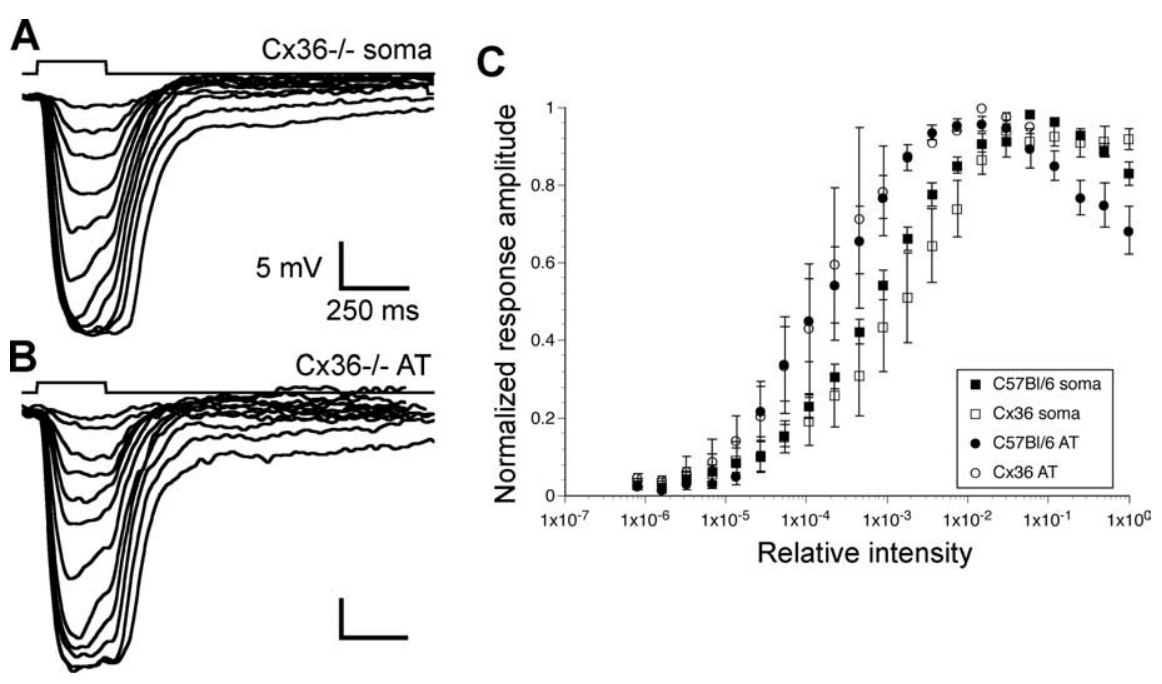

Figure 6. $\quad \boldsymbol{A}, \boldsymbol{B}$, Somatic $(\boldsymbol{A})$ and axon terminal $(\boldsymbol{B})$ recordings from $(x 36$-deficient retinas. Light stimuli are the same as in Figure 2. Both somatic and axon terminal light responses closely resembled responses from wild-type and rhodopsin-deficient retinas, reflecting a strong cone component in both the soma and axon terminal. $\boldsymbol{A}, \boldsymbol{B}$, Calibration: $5 \mathrm{mV}, 250 \mathrm{~ms}$. C, Normalized intensityresponse profiles for C57BL/6 wild-type horizontal cell somata $(n=17)$ and axon terminals $(n=7)$, and Cx36-deficient somata $(n=7)$ and axon terminals $(n=3)$. Error bars indicate mean \pm SE. The intensity that elicited half-maximal responses did not differ significantly between wild-type and (x36-deficient horizontal cell somata or between wild-type and Cx36-deficient horizontal cell axon terminals.

strategy offers the advantage that rod and cone signals can be cleanly isolated; thus, their contributions to wild-type light responses can be accurately assessed.

However, loss of synaptic input commonly causes morphological rearrangement in transgenic mouse retinas (Strettoi et al., 2002; Dick et al., 2003). In particular, deletion of rhodopsin or CNGA3 has been shown to lead to degeneration and/or synaptic rewiring of retinal interneurons (Jaissle et al., 2001; Haverkamp et al., 2006). Nevertheless, detailed morphological studies have determined the time window in which retinas from these mice are fully matured but have not yet started to reorganize; in the present study, care was taken to use mice within this time frame. As an extra precaution, we carefully examined the morphology of the horizontal cells in age-matched mice, using targeted dye injection and immunohistochemistry. We found no signs of degeneration or reorganization in any of the transgenic mouse retinas used in this study (data not shown).

It is possible that, although we detected no morphological changes in the transgenic retinas, functional changes could have taken place. For example, because rods have been shown to have a tonic suppressive effect on cone pathways (Frumkes and Eysteinsson, 1987), one could speculate that loss of rod responses could result in enhancement of cone responses. However, this point was discounted by Williams et al. (2005), who showed that loss of one type of photoreceptor does not effect visual discrimination of the other photoreceptor type.

To provide appropriate controls for both rhodopsin- and CNGA3-deficient mice, we used two different wild-type strains in this study. Interestingly, light responses from C57BL/6 mice differed noticeably in waveform from those of $129 / \mathrm{Sv}$ mice (compare Figs. $2 A, B, 3 A$ ); furthermore, axon terminals from $129 / \mathrm{Sv}$ mice were more sensitive than those of C57BL/6 mice (Figs. $4 D, 5 C$ ). In electroretinograms, a-waves from $\mathrm{C} 57 \mathrm{BL} / 6$ retinas showed a higher sensitivity than those from 129/Sv retinas (Pinto et al., 2007). Thus, the differences seen here probably arise from differences in connectivity between photoreceptors and horizontal cells, rather than from differences in photoreceptor sensitivity. C57BL/6 horizontal cells have larger dendritic fields than those in 129/Sv retinas (Reese et al., 2005), suggesting that they may contact more cones; this could result in a larger suppression of rod inputs than in $129 / \mathrm{Sv}$ retinas.

\section{Rod and cone inputs to horizontal cells}

The horizontal cell axon is believed to electrically isolate the soma from the axon terminal in the mammalian retina. This theory is based on an early study which showed that rod inputs to B-type horizontal cell somata and axon terminals differed in sensitivity; thus, rod signals could not be reaching the soma via the axon terminal (Nelson et al., 1975). Their conclusion was supported by theoretical evidence which suggested that the physical dimensions of the axon were not suited for passive electrical communication between the two cellular compartments (Nelson et al., 1975). It is important to note that their study addressed rod signals, and did not look at how cone inputs arrive at the axon terminals.

In cone horizontal cells in the carp retina, light responses have 

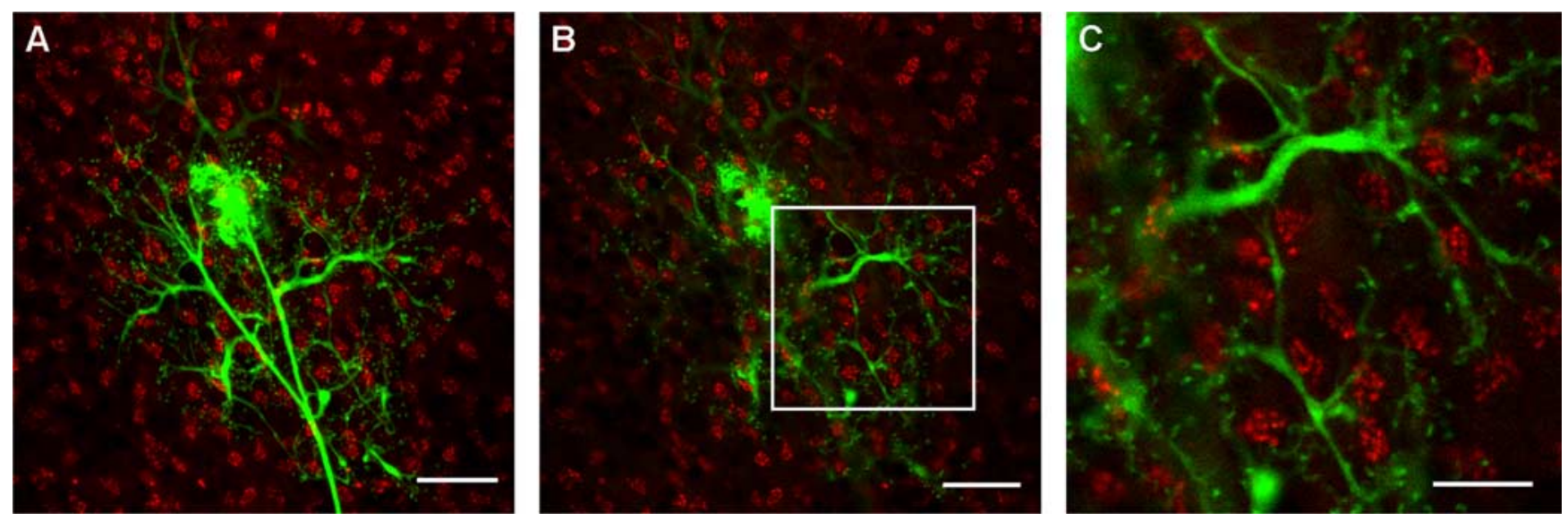

Figure 7. Morphological control. An injected axon terminal (green) from a Cx36-deficient retina labeled with peanut agglutinin (red). No evidence of sprouting of the axon terminal or synaptic contacts with cones was seen. $\boldsymbol{A}$, Image stack. $\boldsymbol{B}$, Single scan. $\boldsymbol{C}$, High magnification of the boxed area in $\boldsymbol{B}$. Scale bars: $\boldsymbol{A}, \boldsymbol{B}, 20 \mu \mathrm{m} ; \boldsymbol{C}, 10 \mu \mathrm{m}$.

Table 1. Rod and cone inputs to somata and axon terminals of the mice used in this study

\begin{tabular}{lll}
\hline & Soma & Axon terminal \\
\hline Wild type & rod + cone & rod + cone \\
Rhodopsin $-1-$ & cone & cone \\
CNGA3 $-1-$ & rod & rod \\
Cx36-1- & $?$ & rod + cone \\
CNGA3 $-1-/$ Cx36-I- & - & rod \\
\hline
\end{tabular}

The photoreceptor contributions to the somatic light responses in the $\mathrm{C} \times 36$-deficient retinas could not be determined because the wild-type light responses were strongly dominated by cone inputs. However, the complete lack of light responses in the somata of $\mathrm{CNGA3} / \mathrm{C} \times 36$ double knock-out mice indicates that rod signals cannot pass to the horizontal cell somata in the absence of rod-cone coupling.

been measured in axon terminals despite the lack of direct photoreceptor inputs to these structures. These light responses were similar in amplitude to those recorded in somata (Weiler and Zettler, 1978; Zettler and Weiler, 1981). It has been suggested that the low conductance of the axon terminal may allow signal conduction from the soma by reducing leakage of signals arriving from the axon (Yagi and Kaneko, 1988). It is not clear whether cone signals pass along the axon to the axon terminal by passive spread (Yagi and Kaneko, 1988), active propagation (Weiler and Zettler, 1978), or gap junctional coupling between axon terminals and the axon near the cell body (Yagi, 1986).

Our findings support the conclusion of Nelson et al. (1975) that rod signals are transmitted to the horizontal cell somata via rod-cone coupling, but suggest that, as seen in fish, cone signals are conducted along the axon from soma to axon terminal. The ion channel composition of the horizontal cell soma differs from that of the axon terminal (A. Feigenspan, unpublished observations). In addition, the channel composition of the axon is not known. Thus, it is possible that specific expression of ion channels in the soma and/or axon allows unidirectional passage of signals from soma to axon terminal.

However, it cannot be ruled out that cone signals reach the axon terminal via gap junctions which do not contain $\mathrm{Cx} 36$. This latter hypothesis suggests that Cx36-containing rod-cone gap junctions are asymmetrical, allowing signal passage from rods to cones but not vice versa. This is supported by expression of Cx36 in cones but not rods in the mouse retina (Feigenspan et al., 2004); these gap junctions are heterotypic. A separate set of gap junctions that allows passage of cone signals into rods could be modulated by different mechanisms, thus allowing distinct regulation of rod-to-cone and cone-to-rod signal spread.

\section{On the distinctive roles of horizontal cell somata and axon terminals}

Interaction between the rod and cone pathways is extensive: rods and cones are electrically coupled (Raviola and Gilula, 1973; Smith et al., 1986; Schneeweis and Schnapf, 1995; Krizaj et al., 1998; Tsukamoto et al., 2001), and rod signals piggyback onto cone pathways at several stages in visual processing (for review, see Völgyi et al., 2004). This interaction of the two photoreceptor systems is essential for adaptation of photoreceptor sensitivity to different levels of ambient illumination. Horizontal cell somata play a key role in this adaptation by averaging light inputs over a large area of retina and feeding this averaged signal back to the cone photoreceptors (Baylor et al., 1971). This feedback adjusts the cone gain to different light levels. It is therefore logical that horizontal cell somata receive inputs from both rod and cone photoreceptors.

It has long been assumed that rod signals reach the horizontal cell somata by way of rod-cone coupling. Raviola and Dacheux (1990) showed that axonless horizontal cells in the rabbit retina receive rod inputs via rod-cone gap junctions. Rod-cone coupling makes up the secondary rod pathway in the mouse retina, and is thought to be mediated by $\mathrm{Cx} 36$ : mice lacking $\mathrm{Cx} 36$ show no intermediate-sensitivity $\mathrm{ON}$ and OFF ganglion cell responses (Deans et al., 2002; Völgyi et al., 2004). For these reasons, we hypothesized that horizontal cell somata in Cx36-deficient mice should not receive rod inputs; this was confirmed in CNGA3Cx36 double knock-out mice. Thus, our data confirm that rods and cones are coupled by $\mathrm{Cx} 36$ in the mouse retina.

Whereas the functional role of the horizontal cell soma is straightforward, the role of the axon terminal is less well understood. Axon terminals have an extraordinarily elaborate morphology (Fig. 7), but no known synaptic output. This structure receives synaptic inputs from the rod photoreceptors, but feedback to the rods has never been demonstrated. Whereas horizontal cell dendrites synapse onto midget bipolar cells in primate cone pedicles (Raviola and Gilula, 1975), axon terminals have not been shown to synapse onto bipolar cells in the mammalian retina. And here we show that axon terminals do not relay signals to the horizontal cell somata.

Horizontal cells have been shown recently to be presynaptic to dopaminergic interplexiform cells in the mouse retina, but it is 
not clear whether the synaptic contacts are made by the axon terminals or the somata (Viney et al., 2007). Our data suggest that horizontal cell axon terminals receive cone signals from the horizontal cell somata; axon terminals therefore receive a mixture of rod and cone signals. These structures could potentially integrate light signals over the entire visual intensity range and over large areas of the retina; they are therefore ideally suited to play a modulatory role in visual processing.

\section{References}

Baylor DA, Fuortes MGF, O’Bryan PM (1971) Receptive fields of cones in the retina of the turtle. J Physiol (Lond) 214:265-294.

Biel M, Seeliger M, Pfeifer A, Kohler K, Gerstner A, Ludwig A, Jaissle G, Fauser S, Zrenner E, Hofmann F (1999) Selective loss of cone function in mice lacking the cyclic nucleotide-gated channel CNG3. Proc Natl Acad Sci U S A 96:7553-7557.

Bloomfield SA, Miller RF (1982) A physiological and morphological study of the horizontal cell types of the rabbit retina. J Comp Neurol 208:288-303.

Deans M, Völgyi B, Goodenough DA, Bloomfield SA, Paul DL (2002) Connexin36 is essential for transmission of rod-mediated visual signals in the mammalian retina. Neuron 36:703-712.

Dick O, tom Dieck S, Altrock WD, Ammermüller J, Weiler R, Garner CC, Gündelfinger ED, Brandstätter JH (2003) The presynaptic active zone protein bassoon is essential for photoreceptor ribbon synapse formation in the retina. Neuron 37:775-786.

Feigenspan A, Janssen-Bienhold U, Hormuzdi S, Monyer H, Degen J, Söhl G, Willecke K, Ammermüller J, Weiler R (2004) Expression of connexin36 in cone pedicles and off-cone bipolar cells of the mouse retina. J Neurosci 24:3325-3334.

Frumkes TE, Eysteinsson T (1987) Suppressive rod-cone interaction in distal vertebrate retina: intracellular records from Xenopus and Necturus. J Neurophysiol 57:1361-1382.

Güldenagel M, Ammermüller J, Feigenspan A, Teubner B, Degen J, Söhl G, Willecke K, Weiler R (2001) Visual transmission deficits in mice with targeted disruption of the gap junction gene connexin36. J Neurosci 21:6036-6044.

Hack I, Peichl L (1999) Horizontal cells of the rabbit retina are nonselectively connected to the cones. Eur J Neurosci 11:2261-2274.

Haverkamp S, Grünert U, Wässle H (2001) The synaptic architecture of AMPA receptors at the cone pedicle of the primate retina. J Neurosci 21:2488-2500.

Haverkamp S, Michalakis S, Claes E, Seeliger MW, Humphries P, Biel M, Feigenspan A (2006) Synaptic plasticity in CNGA3 $(-/-)$ mice: cone bipolar cells react on the missing cone input and form ectopic synapses with rods. J Neurosci 26:5248-5255.

Humphries M, Rancourt D, Farrar J, Kenna P, Hazel M, Bush R, Sieving P, Shiels D, Creighton P, Erven Al, Boros A, Gulya K, Capecchi M, Humphries P (1997) Nat Genet 15:216-219.

Jaissle G, May A, Reinhard J, Kohler K, Fauser S, Lütjen-Drecoll E, Zrenner E, Seeliger M (2001) Evaluation of the rhodopsin knockout mouse as a model of pure cone function. IOVS 42:506-513.

Kolb H (1970) Organization of the outer plexiform layer of the primate retina: electron microscopy of golgi-impregnated cells. Philos Trans R Soc Lond B Biol Sci 258:58261-58283.

Kolb H (1974) The connections between horizontal cells and photoreceptors in the retina of the cat: electron microscopy of Golgi preparations. J Comp Neurol 155:1-14.

Krizaj D, Gábriel R, Owen WG, Witkovsky P (1998) Dopamine D2 receptor-mediated modulation of rod-cone coupling in the Xenopus retina. J Comp Neurol 398:529-538.

Lyubarsky AL, Falsini B, Pennesi ME, Valentini P, Pugh Jr EN (1999) UVand midwave-sensitive cone-driven retinal responses of the mouse: a pos- sible phenotype for coexpression of cone photopigments. J Neurosci 19:442-455.

Nelson R, Lützow AV, Kolb H, Gouras P (1975) Horizontal cells in cat retina with independent dendritic systems. Science 189:137-139.

Niemeyer G, Gouras P (1973) Rod and cone signals in S-potentials of the isolated perfused cat eye. Vision Res 13:1603-1612.

Pinto LH, Invergo B, Shimomura K, Takahashi JS, Troy JB (2007) Interpretation of the mouse electroretinogram. Doc Ophthalmol 115:127-136.

Raviola E, Dacheux RF (1990) Axonless horizontal cells of the rabbit retina: synaptic connections and origin of the rod aftereffect. J Neurocytol 19:731-736.

Raviola E, Gilula NB (1973) Gap junctions between photoreceptor cells in the vertebrate retina. Proc Natl Acad Sci U S A 70:1677-1681.

Raviola E, Gilula NB (1975) Intramembrane organization of specialized contacts in the outer plexiform layer of the retina. J Cell Biol 65:192-222.

Reese BE, Raven MA, Stagg SB (2005) Afferents and homotypic neighbors regulate horizontal cell morphology, connectivity, and retinal coverage. J Neurosci 25:2167-2175.

Schneeweis DM, Schnapf JL (1995) Photovoltage of rods and cones in the macaque retina. Science 268:1053-1056.

Shelley J, Dedek K, Schubert T, Feigenspan A, Hombach S, Willecke K, Weiler $\mathrm{R}$ (2006) Horizontal cell receptive fields are reduced in connexin57deficient mice. Eur J Neurosci 23:3176-3186.

Smith RG, Freed MA, Sterling P (1986) Microcircuitry of the dark-adapted cat retina: functional architecture of the rod-cone network. J Neurosci 6:3505-3517.

Steinberg RH (1969a) Rod and cone contributions to s-potentials from the cat retina. Vision Res 9:1319-1329.

Steinberg RH (1969b) Rod-cone interaction in s-potentials from the cat retina. Vision Res 9:1331-1344.

Steinberg RH (1969c) The rod after-effect in s-potentials from the cat retina. Vision Res 9:1345-1355.

Strettoi E, Porciatti V, Falsini B, Pignatelli V, Rossi C (2002) Morphological and functional abnormalities in the inner retina of the $\mathrm{rd} / \mathrm{rd}$ mouse. J Neurosci 22:5492-5504.

Suzuki H, Pinto LH (1986) Response properties of horizontal cells in the isolated retina of wild-type and pearl mutant mice. J Neurosci 6:1122-1128.

Tsukamoto Y, Morigiwa K, Ueda M, Sterling P (2001) Microcircuits for night vision in mouse retina. J Neurosci 21:8616-8623.

Verweij J, Dacey DM, Peterson BB, Buck SL (1999) Sensitivity and dynamics of rod signals in $\mathrm{H} 1$ horizontal cells of the macaque monkey retina. Vision Res 39:3662-3672.

Viney TJ, Balint K, Hillier D, Siegert S, Boldogkoi Z, Enquist LW, Meister M, Cepko CL, Roska B (2007) Local retinal circuits of melanopsincontaining ganglion cells identified by transsynaptic viral tracing. Curr Biol 17:981-988.

Völgyi B, Deans MR, Paul DL, Bloomfield SA (2004) Convergence and segregation of the multiple rod pathways in mammalian retina. J Neurosci 24:11182-11192.

Weiler R, Zettler F (1978) The axon-bearing horizontal cells in the teleost retina are functional as well as structural units. Vision Res 19:1261-1268.

Williams GA, Daigle KA, Jacobs GH (2005) Rod and cone function in coneless mice. Vis Neurosci 22:807-816.

Witkovsky P, Schmitz Y, Akopian A, Krizaj D, Tranchina D (1997) Gain of rod to horizontal cell synaptic transfer: relation to glutamate release and a dihydropyridine-sensitive calcium current. J Neurosci 17:7297-7306.

Yagi T (1986) Interaction between the soma and the axon terminal of retinal horizontal cells in Cyprinus carpio. J Physiol (Lond) 375:121-135.

Yagi T, Kaneko A (1988) The axon terminal of goldfish retinal horizontal cells: a low membrane conductance measured in solitary preparations and its implication to the signal conduction from the soma. J Neurophysiol 59:482-494.

Zettler F, Weiler R (1981) Propagation of non-spike signals in retinal neurons. Vision Res 21:1589-1590. 